\title{
A NOTE ON $P_{t}$-IDEALS
}

\section{G. T. SPIRASON and E. STRZELECKI}

(Received 29 June 1970)

Communicated by B. Mond

\section{Introduction}

Let $E$ be a vector lattice in the sense of [2]. Two elements $a, b$ in $E$ are said to be disjoint (written $a \perp b$ ) if $|a|^{\wedge}|b|=0$. For any subset $A$ of $E$, $A^{\perp}$ is the set

$$
\{x: x \perp a, \forall a \in A\} .
$$

If $A$ is a one point set $\{a\}$, then we shall use $a^{\perp}$ for $\{a\}^{\perp} \cdot A^{\perp \perp}$ is defined by $A^{\perp \perp}=\left\{A^{\perp}\right\}^{\perp}$. A subset $B$ of $E$ is said to be a polar if it is of the form $A^{\perp}$ for some subset $A$ of $E$. It is well known [1] that the set of all polars of $E$ ordered by inclusion forms a Boolean algebra. We shall denote this algebra by $\mathscr{B}$, and $\mathscr{M}$ will mean the set of all maximal ideals of $\mathscr{B}$. The following properties of polars will be used in the paper.

\section{Proposition 1.1.}

(i) If $A \subseteq B^{\perp}$ then $B^{\perp \perp} \subseteq A^{\perp}$.

(ii) $A^{\perp \wedge} A^{\perp \perp}=E^{\perp}=\{0\}, A^{\perp \vee} A^{\perp \perp}=0^{\perp}=E$ for any $A^{\perp} \in \mathscr{B}$.

(iii) $a \in A^{\perp}$ implies $a^{\perp \perp} \subseteq A^{\perp}$.

(iv) $\bigvee_{\alpha}\left(a_{\alpha}^{\perp \perp}\right)=\left(\bigcup_{\alpha}\left|a_{\alpha}\right|\right)^{\perp \perp}$ whenever $\bigvee_{\alpha}\left|a_{\alpha}\right|$ exists in E.

(v) $a^{\perp \vee} b^{\perp}=\left(|a|^{\wedge}|b|\right)^{\perp}$.

(vi) If $t \in \mathscr{M}$ then for any $A^{\perp} \in \mathscr{B}, t$ contains exactly one of the polars $A^{\perp}$ and $A^{\perp \perp}$.

(vii) If $t \in \mathscr{M}$ then $t$ is prime, i.e. if $A^{\perp \wedge} B^{\perp}=\{0\}$ then $t$ contains at least one of the polars $A^{\perp}$ and $B^{\perp}$.

Properties (i) and (iii) are easily derived. For the proofs of the others we refer to [1] and [4].

Definition 1.2. For $t \in \mathscr{M}$ define $P_{t}$ by

$$
P_{t}=\left\{x: x \in E, x^{\perp \perp} \in t\right\} \text {. }
$$

It is easy to show that $P_{t}$ is a vector lattice ideal of $E$, and that 


$$
E \backslash P_{t}=\left\{y: y^{\perp} \in t\right\} \text {. }
$$

The $P_{t}$-ideals defined above are of special interest; they were considered in [7]. Let

$$
\mathscr{P}=\left\{P_{t}: t \in \mathscr{M}\right\}
$$

we shall sometimes use the expression " $I$ is a $P_{t}$-ideal" instead of writing $I \in \mathscr{P}$.

Theorem 1 gives necessary and sufficient conditions under which a vector lattice ideal $I$ of $E$ belongs to $\mathscr{P}$. Section 2 describes the class of vector lattices $E$ for which every proper $P_{t}$-ideal is minimal prime. For the definitions of a prime vector lattice ideal and a minimal prime ideal we refer to [3].

In the sequel we shall use the properties listed below for prime and minimal prime vector lattice ideals.

Proposition 1.3. The following statements are equivalent.

(i) I is a prime vector lattice ideal of $E$.

(ii) For any $x, y \in E, x \perp y$ implies that $I \cap\{x, y\} \neq \varnothing$.

(iii) For any $y \in E \backslash I, y^{\perp} \subseteq I$.

Proposition 1.4. [3] A prime vector lattice ideal $I$ is minimal if and only if for each $x \in I, x^{\perp} \ddagger I$.

\section{Description of the set $\mathscr{P}$}

THEOREM 1. A vector lattice ideal $I$ of $E$ is a $P_{t}$-ideal (i.e. $I \in \mathscr{P}$ ) if and only if it satisfies the following conditions:

i) I is a prime vector lattice ideal of $E$,

ii) $x \in I \Rightarrow x^{\perp \perp} \subseteq I$,

iii) $x \in I \Rightarrow x^{\perp \perp} \neq E$.

Proof. Assume that $I=P_{t}$ for some $t \in \mathscr{M}$ and suppose that $x \perp y$. Thus $x \in y^{\perp}$ and hence, by 1.1 (i), $y^{\perp \perp} \subseteq x^{\perp}$. Therefore, by 1.1 (ii),

$$
x^{\perp \perp \wedge} y^{\perp \perp} \subseteq x^{\perp \perp \wedge} x^{\perp}=\{0\} \text {. }
$$

According to 1.1 (vii) we conclude that at least one of the polars $x^{\perp \perp}$ and $y^{i \perp}$ belongs to $t$, i.e. $P_{t}$ contains at least one of vectors $x$ and $y$. Thus $P_{t}$ is prime.

Now suppose that $x \in P_{t}$ and $a \in x^{\perp \perp}$. By definition 1.2 we have that $x^{\perp \perp} \in t$. On the other hand $a \in x^{\perp \perp}$ implies, by 1.1 (iii), that $a^{\perp \perp} \subseteq x^{\perp \perp}$, and since $t$ is a lattice ideal containing $x^{\perp \perp}$, this implies that $a^{\perp \perp} \in t$. Thus, by $1.2, a \in P_{t}$. So we have proved that $x^{\perp \perp} \subseteq P_{t}$.

Finally if $x \in P_{t}$ then $x^{\perp \perp} \in t$ and since $t$ is a proper ideal of $\mathscr{B}$ it follows that $x^{\perp \perp} \neq E$.

Conversely, assume that a vector lattice ideal $I$ of $E$ satisfies conditions (i)-(iii) of Theorem 1. Consider the set 


$$
A=\left\{x^{\perp \perp}: x \in I\right\} \bigcup\left\{x^{\perp \perp \vee} y^{\perp}: x \in I, y \notin I\right\} .
$$

The set $A$ has the following properties:

a) The supremum of any two polars in $A$ belongs also to $A$. Indeed, if $x_{1}, x_{2} \in I$ and $y_{1}, y_{2} \notin I$ then according to 1.1 (iv) and the definition of a vector lattice ideal we have

$$
\begin{gathered}
x_{1}^{\perp \perp \vee} x_{2}^{\perp \perp}=\left(\left|x_{1}\right|^{\vee}\left|x_{2}\right|\right)^{\perp \perp} \in A, \\
x_{1}^{\perp \perp \vee}\left(x_{2}^{\perp \perp \vee} y_{1}^{\perp}\right)=\left(\left|x_{1}\right|^{\vee}\left|x_{2}\right|\right)^{\perp \perp \vee} y_{1}^{\perp} \in A, \\
u=\left(x_{1}^{\perp \perp \vee} y_{1}^{\perp}\right)^{\vee}\left(x_{2}^{\perp \perp \vee} y_{2}^{\perp}\right)=\left(\left|x_{1}\right|^{\vee}\left|x_{2}\right|\right)^{\perp \perp \vee}\left(y_{1}^{\perp \vee} y_{2}^{\perp}\right) .
\end{gathered}
$$

But in view of $1.1(\mathrm{v})$,

$$
y_{1}^{\perp \vee} y_{2}^{\perp}=\left(\left|y_{1}\right| \wedge\left|y_{2}\right|\right)^{\perp}
$$

and since $I$ is prime and $y_{1}, y_{2} \notin I$ it follows that $\left|y_{1}\right|^{\wedge}\left|y_{2}\right| \notin I$. Hence the polar $u$ is of the form $x^{\perp \perp \wedge} y^{\perp}$ with $x \in I$ and $y \notin I$ and this belongs also to $A$.

b) $E \notin A$. In fact, if $x \in I$ then by (iii) $x^{\perp \perp} \neq E$. Assume that there exist $x \in I$, $y \notin I$ such that $E=x^{\perp \perp \vee} y^{\perp}$. We have then

$$
y^{\perp \perp}=y^{\perp \perp \wedge} E=\left(x^{\perp \perp^{\vee}} y^{\perp}\right)^{\wedge} y^{\perp \perp}=\left(x^{\perp \perp \wedge} y^{\perp \perp}\right)^{\vee}\{0\}=x^{\perp \perp \wedge} y^{\perp \perp} \text {. }
$$

This means that $y^{\perp \perp} \subseteq x^{\perp \perp}$. Since $y \in y^{\perp \perp}$, it follows that $y \in x^{\perp \perp}$. But, by (ii), $x^{\perp \perp} \subseteq I$ whenever $x \in I$. Thus we conclude that $y \in I$ which contradicts our assumption about $y$.

Properties (a) and (b) imply that the lattice ideal $[A]$ generated by the set $A$ is proper and since $\mathscr{B}$ is a Boolean algebra [1], there exists a maximal ideal $t$ of $\mathscr{B}$ containing $[A]$. We now assert that $I=P_{t}$ for this $t$. In fact, let $x \in I$, then $x^{\perp \perp} \in A \subseteq t$. Thus $x \in P_{t}$. If $y \notin I$ then $y^{\perp}=0^{\perp \perp^{\vee}} y^{\perp} \in A$. Hence $y^{\perp} \in t$ and, by 1.1 (vi), $y^{\perp \perp} \notin t$. So $y \notin P_{t}$.

This concludes the proof of Theorem 1 .

COROLlARY 2.1. $\mathscr{P}$ contains all minimal prime vector lattice ideals of $E$.

Proof. We need only verify that every minimal prime vector lattice ideal $I$ of $E$ satisfies conditions (i)-(iii) of Theorem 1 . Condition (i) is trivially true. Let $x \in E$, then as $I$ is minimal prime, by 1.4 , there exists $y \notin I$ such that $x \perp y$. Since $I$ is prime and $y \notin I$, it follows, by 1.3 , that $y^{\perp} \subseteq I$. But $x \in y^{\perp}$ and hence $x^{\perp \perp} \subseteq y^{\perp}$. Therefore $x^{\perp \perp} \subseteq I$.

Finally, since $y \notin I$, we infer that $y \neq 0$. Thus $y \notin y^{\perp}$ and hence $y^{\perp} \neq E$. The inclusion $x^{\perp \perp} \subseteq y^{\perp}$ implies then that $x^{\perp \perp} \neq E$.

Definition 2.2. An element $e \in E$ is said to be a weak unit if $e^{\perp}=\{0\}$.

It is obvious that $e \in E$ is a weak unit if and only if $e^{\perp \perp}=E$. 
Corollary 2.3. The vector lattice $E$ itself is a $P_{t}$-ideal if and only if $E$ contains no weak units.

Proof. Since $E$ is trivially prime and for any $x \in E, x^{\perp \perp} \subseteq E$, according to Theorem $1, E \in \mathscr{P}$ if and only if for any $x \in E, x^{\perp \perp} \neq E$. Thus $E \in \mathscr{P}$ if and only if no $x \in E$ is a weak unit.

Corollary 2.3 shows that a $P_{t}$-ideal is not necessarily minimal prime. In fact, consider the following.

EXAMPLE 1. Let $L$ be the vector lattice of sequences $\left\{\alpha_{n}\right\}$ of real numbers such that for each $\left\{\alpha_{n}\right\}$ only a finite number of its terms differs from zero. Define $\left\{\alpha_{n}\right\} \geqq 0$ if and only if $\alpha_{n} \geqq 0$ for each $n$. The vector lattice $L$ has no weak unit and thus $L \in \mathscr{P}$.

The next example describes a vector lattice $E$ having a proper $P_{t}$-ideal which s not minimal prime.

Example 2. Let $E=\left\{\left(\gamma,\left\{\alpha_{n}\right\}\right): \gamma \in R,\left\{\alpha_{n}\right\} \in L\right\}$, where $L$ is the vector lattice of example 1. Define the order in $E$ by $\left(\gamma,\left\{\alpha_{n}\right\}\right) \geqq 0$ if and only if either $\gamma>0$ or $\gamma=0$ and $\alpha_{n} \geqq 0$ for all $n$. $E$ has the following properties:

i) $E$ is a vector lattice.

ii) $\left|\left(\gamma,\left\{\alpha_{n}\right\}\right)\right|=\left\{\begin{array}{l}\left(\gamma,\left\{\alpha_{n}\right\}\right) \text { if } \gamma>0 \\ \left(-\gamma,\left\{-\alpha_{n}\right\}\right) \text { if } \gamma<0 \\ \left(0,\left\{\left|\alpha_{n}\right|\right\}\right) \text { if } \gamma=0 .\end{array}\right.$

iii) If $\left(\gamma,\left\{\alpha_{n}\right\}\right) \neq 0$ then

$$
\left(\gamma,\left\{\alpha_{n}\right\}\right)^{\perp}=\left\{\begin{array}{l}
\{0) \text { if } \gamma \neq 0 \\
\left\{\left(0,\left\{\beta_{n}\right\}\right):\left\{\beta_{n}\right\} \in L, \alpha_{n} \beta_{n}=0 \forall n\right\} \text { if } \gamma=0 .
\end{array}\right.
$$

iv) The set $I=\left\{\left(0,\left\{\alpha_{n}\right\}\right):\left\{\alpha_{n}\right\} \in L\right\}$ is a proper vector lattice ideal of $E$.

v) $I$ satisfies all conditions of Theorem 1 and thus $I \in \mathscr{P}$.

vi) $I$ is not minimal because if $\left(0,\left\{\alpha_{n}\right\}\right) \neq 0$ then $\left(0,\left\{\alpha_{n}\right\}\right)^{\perp} \subseteq I$.

In section 3 we describe the class of vector lattices for which every proper $P_{t}$-ideal is minimal prime. It turns out that this class is quite large, and contains many important types of vector lattices.

\section{Weakly projectable vector lattices}

Definition 3.1. A vector lattice $E$ is said to be weakly projectable if for any $x, y \in E$ there exists $z \in x^{\perp}$ such that $y \in(|x|+|z|)^{\perp \perp}$.

THEOREM 2. Each proper $P_{t}$-ideal of a vector lattice $E$ is minimal prime if and only if $E$ is weakly projectable.

Proof. Suppose that $E$ is weakly projectable and that $P_{t} \in \mathscr{P}$ is not minimal 
prime. Then, by 1.4 , there exists $a \in P_{t}$ such that $a^{\perp} \subseteq P_{t}$. According to definition 3.1, for any $x \in E$ there exists $z \in a^{\perp}$ such that

$$
x \in(|a|+|z|)^{\perp \perp} \text {. }
$$

But $a \in P_{t}$ and $z \in a^{\perp} \subseteq P_{t}$. Thus $|a|+|z| \in P_{t}$. Consequently, by Theorem 1,

$$
(|a|+|z|)^{\perp \perp} \subseteq P_{t} \text {. }
$$

Since $x \in(|a|+|z|)^{\perp \perp}$ it follows that $x \in P_{t}$, i.e. $P_{t}=E$.

Conversely, assume that $E$ is not weakly projectable. Then there exists $a, b$ in $E$ such that whenever $z \in a^{\perp}$,

$$
b \notin(|a|+|z|)^{\perp \perp} .
$$

Now consider the set $B$ of polars defined by

$$
B=\left\{(|a|+|z|)^{\perp \perp{ }^{*}} b^{\perp}: z \in a^{\perp}\right\} .
$$

Since $b \notin(|a|+|z|)^{\perp \perp}$, it follows that $b^{\perp \perp} \ddagger(|a|+|z|)^{\perp \perp}$. Thus,

$$
\begin{aligned}
\left((|a|+|z|)^{\perp \perp^{\vee}} b^{\perp}\right)^{\wedge} b^{\perp \perp} & =\left((|a|+|z|)^{\perp \wedge \wedge} b^{\perp \perp}\right)^{\vee}\left(b^{\perp \wedge} b^{\perp \perp}\right) \\
& =(|a|+z \mid)^{\perp \perp \wedge} b^{\perp \perp} \neq b^{\perp \perp .}
\end{aligned}
$$

This means that for all $z \in a^{\perp},(|a|+|z|)^{\perp \perp^{\vee}} b^{\perp} \neq E$. Now, since $z \perp a$, $|a|+|z|=|a|^{\vee}|z|$. Then by 1.1 (iv),

$$
(|a|+|z|)^{\perp \perp \vee} b^{\perp}=\left(|a|^{\vee}|z|\right)^{\perp \perp \vee} b^{\perp}=|a|^{\perp \perp \vee}|z|^{\perp \perp \vee} b^{\perp} .
$$

Thus for $z_{1}, z_{2} \in a^{\perp}$ we have

$$
\begin{aligned}
u & =\left(\left(|a|+\left|z_{1}\right|\right)^{\perp \perp \wedge} b^{\perp}\right)^{\vee}\left(\left(|a|+\left|z_{2}\right|\right)^{\perp \perp \vee} b\right) \\
& =|a|^{\perp \perp \vee}\left|z_{1}\right|^{\perp \wedge}\left|z_{2}\right|^{\perp \perp \vee} b^{\perp} \\
& =|a|^{\perp \perp \vee}\left(\left|z_{1}\right|^{\vee}\left|z_{2}\right|\right)^{\perp \perp^{\vee}} b^{\perp} .
\end{aligned}
$$

But $z_{1}, z_{2} \in a^{\perp}$ implies that $z_{3}=\left|z_{1}\right|^{\vee}\left|z_{2}\right| \in a^{\perp}$ and $\left|z_{3}\right|=z_{3}$. Therefore

$$
u=|a|^{\perp \perp \vee}\left|z_{3}\right|^{\perp \perp^{\vee}} b^{\perp}=\left(|a|+\left|z_{3}\right|\right)^{\perp \nu^{\vee}} b^{\perp} \in B \text {. }
$$

Since no polar in $B$ is the whole space, and the supremum of any two polars in $B$ belongs to $B$, the lattice ideal $[B]$ generated by $B$ is proper. Thus there exists a maximal ideal $t$ of $\mathscr{B}$ containing $B$. Consider $P_{t}$ defined by this $t$. Since $b^{\perp}$ is contained in each polar of $B$ we conclude that $b^{\perp} \in t$ and so $b^{\perp \perp} \notin t$. This means that $b \notin P_{t}$, and consequently, $P_{t}$ is proper. On the other hand repeating the arguments presented in the proof of the second part of Theorem 1 we infer that $a \in P_{t}$ and $a^{\perp} \subseteq P_{t}$, hence $P_{t}$ is not minimal. 
This concludes the proof of Theorem 2. Combining Corollary 2.3 with Theorem 2 we obtain

Corollary 3.2. All $P_{t}$-ideals of a vector lattice $E$ are minimal if and only if $E$ is weakly projectable and possesses a weak unit.

Example 2 of the preceding section shows that not all vector lattices are weakly projectable. The space considered there was non-archimedean. The authors were unable until now to provide an example of an Archimedean vector lattice which is not weakly projectable.

The following shows that the class of weakly projectable spaces is quite large.

Definition 3.3 [5]. A vector lattice $E$ is said to be Stone if for each $a \in E$ we have $E=a^{\perp} \oplus a^{\perp \perp}$.

THEOREM 3. Every Stone vector lattice is weakly projectable.

Proof. Since $E$ is Stone, for any $x, y \in E$ there exist $y_{1} \in x^{\perp \perp}$ and $y_{2} \in x^{\perp}$ such that $y=y_{1}+y_{2}$. Thus we have that

$$
\begin{aligned}
y \in\left(y_{1}+y_{2}\right)^{\perp \perp} & =\left(\left|y_{1}+y_{2}\right|\right)^{\perp \perp} \subseteq\left(\left|y_{1}\right|+\left|y_{2}\right|\right)^{\perp \perp} \\
& =\left(\left|y_{1}\right|^{\vee}\left|y_{2}\right|\right)^{\perp \perp}=\left|y_{1}\right|^{\perp \vee}\left|y_{2}\right|^{\perp \perp} \subseteq|x|^{\perp \perp \vee}\left|y_{2}\right|^{\perp \perp} \\
& =\left(|x|+\left|y_{2}\right|\right)^{\perp \perp},
\end{aligned}
$$

where $y_{2} \in x^{\perp}$.

REMARK. The last inclusion follows from the fact that $y_{1} \in x^{\perp .1}$ implies that $\left|y_{1}\right|^{\perp \perp} \subseteq|x|^{\perp \perp}$.

Obviously every Riesz space (space with projections) is Stone. According to [6], every $\sigma$-complete space is a Stone space. Thus the class of weakly projectable vector lattices contains all the above-mentioned types of vector lattices. On the other hand it is essentially larger. It was shown in [5] that the vector lattice $C[0,1]$ of all continuous real valued functions defined on the interval $[0,1]$ was not Stone. However, the following holds:

Proposition 3.4. $C[0,1]$ is weakly projectable.

Proof. For $x \in C[0,1]$, define $S_{x}$ by

$$
S_{x}=\{t: t \in[0,1], x(t) \neq 0\},
$$

and $N_{x}$ by $N_{x}=[0,1] \backslash S_{x}$. First of all let us note that for any open subset $G$ of $[0,1]$ there exists a $z \in C[0,1]$ such that $S_{z}=G$. Indeed define the function $z$ as the distance to the nearest point of the complement of $G$ except that if there is no such point (if $G$ is the whole space) then take $z(t) \equiv 1$ for $\forall t$. 
Given an element $x$ of the space, let $A$ be the interior of $N_{x}$. Choose $z \in C[0,1]$ to have the property $S_{z}=A$. Suppose that

$$
y \perp(|x|+|z|), y \in C[0,1] .
$$

Since $y \perp|x|, S_{y}$ is an open set disjoint from $S_{x}$, i.e. $S_{y} \subseteq A$. But $y \perp|z|$ implies that

$$
S_{y}=S_{y} \cap A=\varnothing .
$$

Thus $N_{y}=[0,1]$, i.e. $y(t)=0$ for all $t \in[0,1]$. Consequently,

$$
(|x|+|z|)^{\perp}=\{0\}
$$

and hence $(|x|+|z|)^{\perp \perp}=C[0,1]$. This means that $C[0,1]$ is weakly projectable.

\section{References}

[1] S. J. Bernau, 'Unique representations of Archimedean lattice groups and normal Archimedean lattice rings', Proc. Lond. Math. Soc. (Series 3), Vol. 15 (1965), 599-630.

[2] G. Birkhoff, Lattice Theory, 3rd edition (American Math. Soc., Providence, Rhode Island, 1967).

[3] D. G. Johnson and J. E. Kist, 'Prime ideals in vector lattices', Can. J. Math. Vol. 14 (1962), 517-528.

[4] R. Sikorski, Boolean Algebras (2nd edition, Springer-Verlag, 1964).

[5] T. P. Speed and E. Strzelecki, 'A note on commutative $\mathscr{L}$-groups', (To appear).

[6] T. P. Speed, 'On commutative $\mathscr{L}$-groups', (To appear).

[7] A. J. Veksler, 'Linear spaces with disjoint elements and their conversion into vector lattices', Leningrad Gos. Ped. Inst. Učen. Zap. 328 (1967), 19-43.

\section{Monash University}

Victoria, 3168

Australia. 